\title{
Índice de envejecimiento activo en Colombia: análisis basado en la Encuesta Nacional de Salud, Bienestar y Envejecimiento (SABE Colombia 2015)
}

\author{
Francisco J. Tamayo Giraldo', Lindi I. Baracaldo Pinzón², Sandra L. Valencia Almonacid³, Delia Ortega \\ Lenis $^{1}$ y Margarita M. Giraldo Cárdenas ${ }^{4}$
}

Forma de citar Tamayo Giraldo FJ, Baracaldo Pinzón LI, Valencia Almonacid SL, Ortega Lenis D, Giraldo Cárdenas MM. Índice de enveje-
cimiento activo en Colombia: análisis basado en la Encuesta Nacional de Salud, Bienestar y Envejecimiento (SABE Colombia
2015). Rev Panam Salud Publica. 2021;45:e69. https://doi.org/10.26633/RPSP.2021.69

RESUMEN

Objetivos. Describir el estado actual de la población de personas de 60 años o más de Colombia, y determinar el índice de envejecimiento activo (IEA) y sus dominios.

Métodos. A partir de los datos recolectados durante la Encuesta Nacional de Salud, Bienestar y Envejecimiento realizada en Colombia en 2015 (SABE Colombia 2015), se calculó el IEA para Colombia, según la metodología elaborada para calcular el IEA para los países de la Unión Europea y sus cuatro dominios, adaptados a los datos que ofrece la encuesta y el contexto colombianos.

Resultados. El IEA para Colombia es de 37,4. Se observó una alta empleabilidad en las personas mayores colombianas, pero el mayor porcentaje de empleos se encuentra dentro del sector informal. Un bajo porcentaje de las personas mayores tiene actividades en grupos políticos, sindicales o que impliquen tomar decisiones de impacto para la sociedad y el país. El hecho de que la gran mayoría de las personas mayores de Colombia tiene una adecuada funcionalidad, abre las posibilidades para elevar su desempeño de acuerdo con los determinantes del envejecimiento activo.

Conclusiones. Se presenta una primera aproximación diagnóstica real de la situación de las personas mayores en Colombia y sus posibilidades de transitar por un envejecimiento activo. Se deben modificar las políticas públicas para cerrar las brechas identificadas y elevar la calidad de vida de las personas mayores mediante la oferta de mejores oportunidades que les permitan optar por una adecuada remuneración y una mejor calidad de vida. Se debe cambiar de una visión de servicios centrados en la enfermedad a nuevos enfoques basados en la promoción de la salud y la prevención de la discapacidad.

Palabras clave Anciano; envejecimiento saludable; Colombia.

La Organización Mundial de la Salud (OMS) define el envejecimiento saludable como "el proceso de fomentar y mantener la capacidad funcional que permite el bienestar en la vejez" (1). Con la intención de mover el foco de atención hacia aspectos más puntuales del envejecimiento poblacional, la OMS introdujo a finales del siglo XX el concepto de envejecimiento activo, definido como "el proceso por el cual se optimizan las oportunidades de bienestar físico, social y mental durante toda la vida con el objetivo de ampliar la esperanza de vida saludable, la productividad y la calidad de vida en la vejez" $(2,3)$. Este concepto abarca también a las personas discapacitadas, de frágil salud o que necesitan asistencia, ya que todos pueden ayudar a

\footnotetext{
1 Pontificia Universidad Javeriana, Cali, Colombia $\triangle$ Francisco J. Tamayo Giraldo, franciscoj.tamayo@javerianacali.edu.co

2 Secretaria de Salud, Villavicencio, Colombia.
}

\footnotetext{
3 Secretaría de Salud, Cali, Colombia.

4 Clínica Farallones, Cali, Colombia.
}

Este es un artículo de acceso abierto distribuido bajo los términos de la licencia Creative Commons Attribution-NonCommercial-NoDerivs 3.0 IGO, que permite su uso, distribución y reproducción en cualquier medio, siempre que el trabajo original se cite de la manera adecuada. No se permiten modificaciones a los articulos ni su uso comercial. Al reproducir un artículo no debe haber ningún indicio de que la OPS o el artículo avalan a una organización o un producto específico. El uso del logo de la OPS no está permitido. Esta leyenda debe conservarse, junto con la URL original del artículo. Crédito del logo y texto open access: PLoS, bajo licencia Creative Commons Attribution-Share Alike 3.0 Unported. 
sus semejantes y contribuir activamente con sus comunidades, sociedades y países $(4,5)$.

En el año 2002 se llevó a cabo en Madrid la Segunda Asamblea Mundial sobre Envejecimiento, convocada por las Naciones Unidas, con el fin de actualizar el plan de acción internacional e invitar a los países en desarrollo a asumir compromisos que permitieran iniciar y fortalecer las iniciativas gubernamentales y no gubernamentales tendientes a mejorar el bienestar de las personas mayores. El marco establecido abarca tópicos de vital importancia, como el envejecimiento y la pobreza, el envejecimiento y las políticas de salud, la influencia de la vida productiva en el envejecimiento, el papel y la cooperación de los sectores público y privado, las perspectivas gerontológicas y geriátricas, los derechos de los adultos mayores, la discriminación de género, el envejecimiento rural, las relaciones intergeneracionales, la vivienda, la familia, la comunidad y las redes sociales, entre otros $(6,7)$. En esa ocasión se definió la necesidad de afrontar el envejecimiento poblacional, enfocados en el envejecimiento activo y sobre tres pilares: la participación, la salud y la seguridad (3). De igual manera, se decidió que los países de las Américas realizarían conferencias regionales periódicas con el fin de evaluar y hacer el seguimiento de los avances logrados, proceso que llevó a formular en 2015 la Declaración de los Derechos Humanos de las Personas Mayores, ratificada en la Cuarta Conferencia Regional Intergubernamental sobre Envejecimiento y Derechos de las Personas Mayores en América Latina y el Caribe, celebrada en Asunción, Paraguay, en 2017 (8).

En Colombia, se han logrado avances en materia de legislación y políticas públicas, materializados en la aprobación de la Política de Envejecimiento Humano y Vejez 2014-2024 (9), fundamentada en cuatro ejes:

- Eje 1: Promoción y garantía de los derechos humanos de las personas mayores

- Eje 2: Protección social integral

- Eje 3: Envejecimiento activo, satisfactorio y saludable

- Eje 4: Formación del talento humano e investigación en esta materia.

El eje 3 se incluyó en el Plan Decenal de Salud Pública 2012-2021.

En el 2012, en el marco del décimo aniversario de la Segunda Asamblea Mundial de Envejecimiento, se presentó el índice de envejecimiento activo (IEA) para los países de la Unión Europea (10). Este índice mide el nivel de vida independiente que tienen las personas mayores, su participación en el trabajo remunerado y en actividades sociales, así como su capacidad para mantenerse activas en la vejez.

En Colombia no se cuenta aún con una aproximación cuantitativa y objetiva del envejecimiento activo, pero es posible estimarla a partir de los datos recabados en 2015 durante la Encuesta Nacional de Salud, Bienestar y Envejecimiento (SABE Colombia 2015) — llevada a cabo por el Sistema Nacional de Estudios y Encuestas Poblacionales en Salud, del Ministerio de Salud y Protección Social-, cuyo marco conceptual está basado en determinantes que permitirían estimar el IEA para Colombia (11). La información que proporciona este índice podría ayudar en la identificación de lagunas y la implementación de estrategias informadas que promuevan el envejecimiento activo en todo el país, con políticas que no se orienten solamente a elevar el bienestar de las personas mayores, sino que también tomen en cuenta el aporte de este sector de la población a la economía y la sociedad.

En este artículo se describe el estado actual de la población de personas de 60 años o más de Colombia, y se determina el IEA y sus dominios a partir de los datos recolectados durante la encuesta SABE Colombia 2015.

\section{MATERIALES Y MÉTODOS}

Se realizó un estudio cuantitativo, analítico de corte transversal a partir de la información contenida en la base de datos de la encuesta SABE Colombia 2015. Esta encuesta se basó en un muestreo probabilístico, estratificado y por conglomerados con representatividad nacional, regional, departamental y de las grandes ciudades (Cali, Medellín y Barranquilla; Bogotá se clasificó como una región) de personas de 60 años o más. Se encuestaron 23694 personas (77,1\% de la muestra prevista); a 19003 (80,2\%) de ellos se les aplicó la encuesta directamente y a 4691 (19,8\%) a través de un familiar cercano o cuidador. Los detalles metodológicos y sus resultados se encuentran ya publicados (11).

Se calculó el IEA para Colombia según la metodología empleada para el cálculo del IEA europeo (10), ajustada a los datos colectados en la encuesta SABE Colombia 2015. Para el dominio actividades remuneradas se utilizó la variable "empleo", y para el dominio actividades no remuneradas se empleó la variable "participación social" (en grupos religiosos, deportivos, políticos, culturales, comunitarios, ecológicos, gremiales, étnicos, de salud y de personas mayores en general). El color de la piel se clasificó según la paleta de colores usada en el barómetro de las Américas 2010 (12). Además se analizaron los dominios vida autónoma e independiente, y entorno propicio y saludable para el envejecimiento activo (figura 1).

Para la construcción del IEA de Colombia se mantuvieron los principios adoptados en la metodología europea:

1. Los indicadores del índice se expresaron en forma positiva.

2. Los indicadores se enunciaron en términos porcentuales (de $0 \%$ a $100 \%$ ).

3. Se calculó el porcentaje para cada indicador y para cada dominio.

4. El IEA para Colombia se calculó como la suma aritmética de los índices ponderados de cada dominio.

Se empleó el valor de la expectativa de vida a los 60 años en Colombia de 24,04 años, según el estimado realizado por el Departamento Nacional de Estadística (DANE) en 2015 (14); para calcular la proporción de la esperanza de vida alcanzada sobre el objetivo de vivir 105 años en el dominio entorno propicio y saludable para el envejecimiento activo, se dividió ese valor entre 60. Otras variables para las que se utilizaron los datos del DANE fueron: el promedio de ingresos en personas de 60 años o más, a partir de los datos de ingreso per cápita en Colombia; la proporción de mayores de 65 años que fungen como cabeza de hogares que se encuentran por encima del nivel de pobreza; y la proporción de la esperanza de vida saludable después de los 60 años sobre la esperanza de vida a esa edad.

\section{Análisis de los datos}

Para la creación de cada indicador se revisaron las preguntas de la encuesta SABE Colombia 2015, según la definición del IEA 
FIGURA 1. Variables empleadas para estimar el índice de envejecimiento activo para Colombia según los dominios analizados
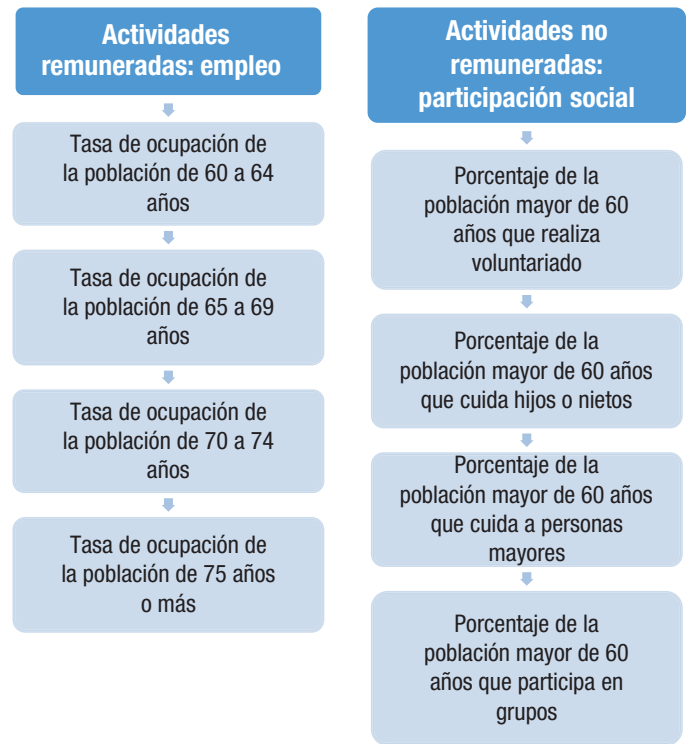

Fuente: elaborada por los autores.

Fura determinar funcionalidad en personas mayores (13).

Calculado como el valor de la esperanza de vida a los 60 años en Colombia (24,04 años) dividido entre 60 años.

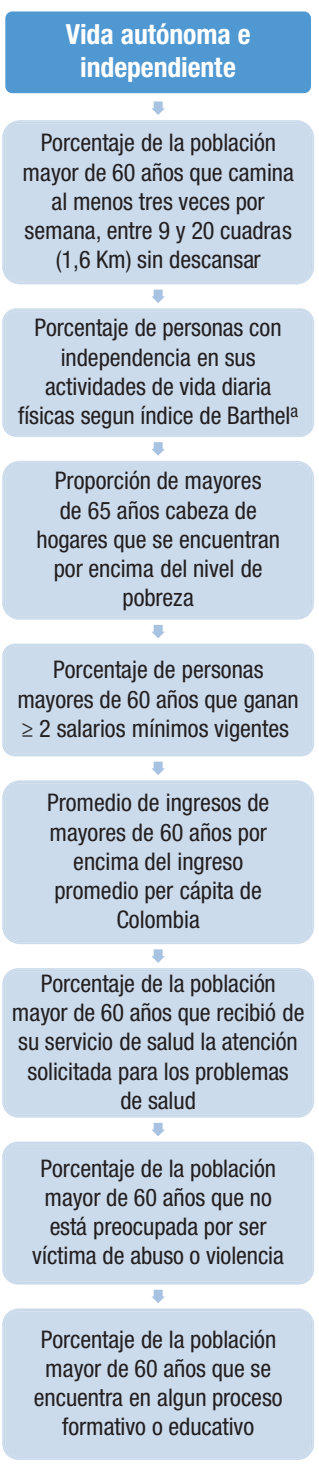

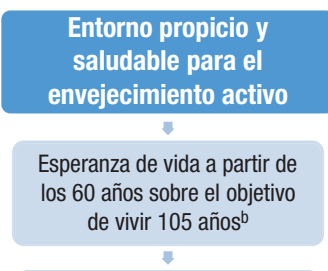

Proporción de esperanza de vida saludable despues de los 60 años sobre esperanza de vida a los 60 años

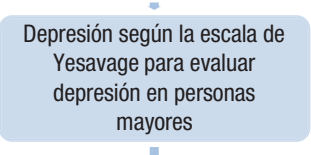

Porcentaje de la población mayor de 60 años que utiliza computador o Internet al menos 1 vez por semana

Porcentaje de la población mayor de 60 años que recibe cariño y afecto de sus hijos

Porcentaje de la población mayor de 60 años que recibe cariño y afecto de los convivientes

Porcentaje de la población
mayor de 60 años que recibe
cariño y afecto de los amigos
Porcentaje de la población
mayor de 60 años con
educación secundaria o
superior

empleada en la metodología europea, y se agruparon los resultados según los dominios de ese índice. Para cada una de las variables identificadas (figura 1) se realizó un análisis descriptivo mediante la estimación de proporciones por cada categoría con sus respectivos intervalos de confianza de 95\% (IC95\%).

Los índices específicos para cada uno de los cuatro dominios se calcularon como la media aritmética ponderada de sus indicadores, con los pesos de ponderación recomendados en la metodología utilizada por la Comisión Económica de las Naciones Unidas para Europa (10): 35\% para los dos primeros dominios, $10 \%$ para el tercero y $20 \%$ para el cuarto. Igualmente, se ponderaron los porcentajes de cada variable (cuadro 1 ).

Para el procesamiento de los datos y su análisis se utilizó el paquete estadístico IBM-SPSS versión 25.

Este proyecto recibió la aprobación del Comité de Ética en Investigación de la Facultad de Ciencias de la Salud de la Pontificia Universidad Javeriana de Cali, Colombia. La base de datos se empleó con el permiso otorgado por el Ministerio de Salud y Protección Social de Colombia. Para proteger la identidad de los participantes se codificaron las variables que lo requerían.

\section{RESULTADOS}

\section{Variables sociodemográficas}

Se observó una mayor proporción de mujeres que de hombres en el total nacional de personas mayores, especialmente en las grandes ciudades, donde la relación alcanzó valores de 110 a 140 mujeres por cada 100 hombres; en zonas urbanas residía entre el 77,0\% y el 80,6\% de las personas mayores encuestadas. Con respecto al color de la piel de los participantes, $54,2 \%$ de los encuestados se consideraron como de piel clara, valor que ascendió a 59,0\% en las mujeres. 
CUADRO 1. Índice de envejecimiento activo (IEA) para Colombiaa , según la ponderación porcentual de dominios y variables

\begin{tabular}{|c|c|c|c|c|c|c|}
\hline Dominio (peso total del dominio ${ }^{b}$ ) & Variable, según SABE Colombia 2015 & $\begin{array}{l}\text { Peso de la } \\
\text { variable }^{\mathrm{b}}\end{array}$ & IEA & $\begin{array}{l}\text { Ponderación } \\
\text { por el } \\
\text { número de } \\
\text { variables }\end{array}$ & $\begin{array}{c}\text { IEA } \\
\text { ponderado }\end{array}$ & $\begin{array}{l}\text { IEA del } \\
\text { dominio }\end{array}$ \\
\hline \multirow{3}{*}{$\begin{array}{l}\text { 1. Actividades remuneradas: } \\
\text { empleo }(0,35)\end{array}$} & De 65 a 69 años & 25 & 45,1 & 0,25 & 11,2 & \multirow{3}{*}{17,4} \\
\hline & De 70 a 74 años & 25 & 36,6 & 0,25 & 9,1 & \\
\hline & 75 años o más & 25 & 61,3 & 0,25 & 15,3 & \\
\hline \multirow{2}{*}{$\begin{array}{l}\text { 2. Actividades no remuneradas: } \\
\text { participación social en mayores } \\
\text { de } 60 \text { años }(0,35)\end{array}$} & Cuidan de personas mayores (\%) & 30 & 2,5 & 0,3 & 0,7 & \multirow{2}{*}{10,1} \\
\hline & Participan en grupos (\%) & 20 & 46,5 & 0,2 & 9,3 & \\
\hline \multirow[t]{6}{*}{$\begin{array}{l}\text { 3. Vida autónoma e independiente } \\
\text { en mayores de } 60 \text { años }(0,1)\end{array}$} & $\begin{array}{l}\text { Caminan al menos tres veces por semana, entre } 9 \text { y } 20 \text { cuadras } \\
(1,6 \mathrm{~km}) \text { sin descansar }(\%)\end{array}$ & 10 & 52,5 & 0,1 & 5,2 & \multirow[t]{6}{*}{5,9} \\
\hline & $\begin{array}{l}\text { Recibió de su servicio de salud la atención solicitada para los } \\
\text { problemas de salud (\%) }\end{array}$ & 20 & 92,3 & 0,2 & 18,4 & \\
\hline & Ganan $\geq 2$ salarios mínimos vigentes $(\%)$ & 10 & 9,4 & 0,1 & 0,94 & \\
\hline & $\begin{array}{l}\text { Proporción de personas con } 60 \text { años o más con ingresos } \\
\text { superiores al promedio per cápita en Colombia }\end{array}$ & 10 & 0,3 & 0,1 & 0,03 & \\
\hline & No reportan riesgo de abuso o violencia (\%) & 10 & 96,2 & 0,1 & 9,6 & \\
\hline & Actualmente se encuentran estudiando (\%) & 10 & 0,9 & 0,1 & 0,09 & \\
\hline \multirow{4}{*}{$\begin{array}{l}\text { 4. Entorno propicio y saludable para } \\
\text { el envejecimiento activo en mayores } \\
\text { de } 60 \text { años }(0,2)\end{array}$} & $\begin{array}{l}\text { Proporción de la esperanza de vida alcanzada sobre el objetivo } \\
\text { de vivir } 105 \text { años }\end{array}$ & 33,3 & 0,40 & 0,33 & 0,13 & \multirow[t]{4}{*}{4,0} \\
\hline & $\begin{array}{l}\text { Proporción de esperanza de vida saludable después de los } 60 \\
\text { años sobre la esperanza de vida a los } 60 \text { años }\end{array}$ & 23,3 & 0,03 & 0,24 & 0,01 & \\
\hline & Sin depresióne (\%) & 16,7 & 42,7 & 0,16 & 7,13 & \\
\hline & $\begin{array}{l}\text { Utilizan Internet o computador por lo menos una vez a la semana } \\
\text { para sus actividades personales (\%) }\end{array}$ & 6,7 & 7,4 & 0,07 & 0,5 & \\
\hline
\end{tabular}

Fuente: elaborada por los autores.

aEl índice de envejecimiento activo para Colombia es 37,4 .

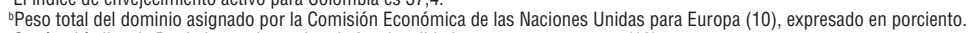

'Según el índice de Barthel para determinar la funcionalidad en personas mayores (13).

${ }^{\circledR}$ Calculado como el valor de la esperanza de vida a los 60 años en Colombia (24,04 años) dividido entre 60 años.

eSegún la escala de Yesavage para evaluar la depresión en personas mayores.

La mayor proporción de personas mayores correspondió a la región central $(27,1 \%$ de la población), mientras que en Orinoquia-Amazonia se encontró el menor porcentaje $(1,4 \%)$. En general, predominaron las personas de 60 a 69 años $(57,2 \%)$. El $54,2 \%$ se reconoció como de piel clara, el $34,7 \%$ como de piel con color medio (ni blanco ni negro) y el 11,1\% como de piel oscura (cuadro 2); más de la mitad de las personas que se consideraron de piel oscura eran hombres $(54,2 \%)$, mientras que la mayoría de las personas con piel clara eran mujeres $(59,0 \%)$.

\section{Actividades remuneradas y empleo}

El $56,6 \%$ de los hombres y el 28,2\% de las mujeres ejercían un oficio, pero solo el $42,2 \%$ recibía alguna remuneración por ello; sin embargo, el $67,4 \%$ de los encuestados que no trabajaban estaban afiliados al sistema contributivo, en tanto que el 74,5\% de quienes estaban afiliados a regímenes especiales no tenían una ocupación. Del total de los no afiliados al sistema de salud, el 61,4\% tenía alguna ocupación o trabajo.

\section{Actividades no remuneradas y participación social}

El 46,5\% de los encuestados formaba parte de al menos un grupo de personas como parte de su actividad social; de ese total, el 49,7\% eran mujeres. La mayor participación se observó en las personas de 65 a 69 años (47,9\%). Significativamente más mujeres (62,9\%; IC95\%: 62,0-63,8) que hombres (54,4\%; IC95\%: $53,4-55,5)$ participaban en el cuidado de los nietos.

El 84,0\% de los encuestados no hacían actividades de voluntariado: solo el $15,4 \%$ de los hombres y el $16,5 \%$ de las mujeres lo hacían.

\section{Vida autónoma y entorno propicio para el envejecimiento activo}

El 79,1\% (IC95\%: 78,6-79,7) de los encuestados no hacía deportes en grupos organizados, aunque el 52,5\% (IC95\%: 51,853,2 ) informó caminar $1,6 \mathrm{~km}$ al menos tres veces por semana. El $92,3 \%$ de las personas que respondieron la encuesta recibieron 
CUADRO 2. Distribución de las personas mayores según variables sociodemográficas, Colombia, 2015

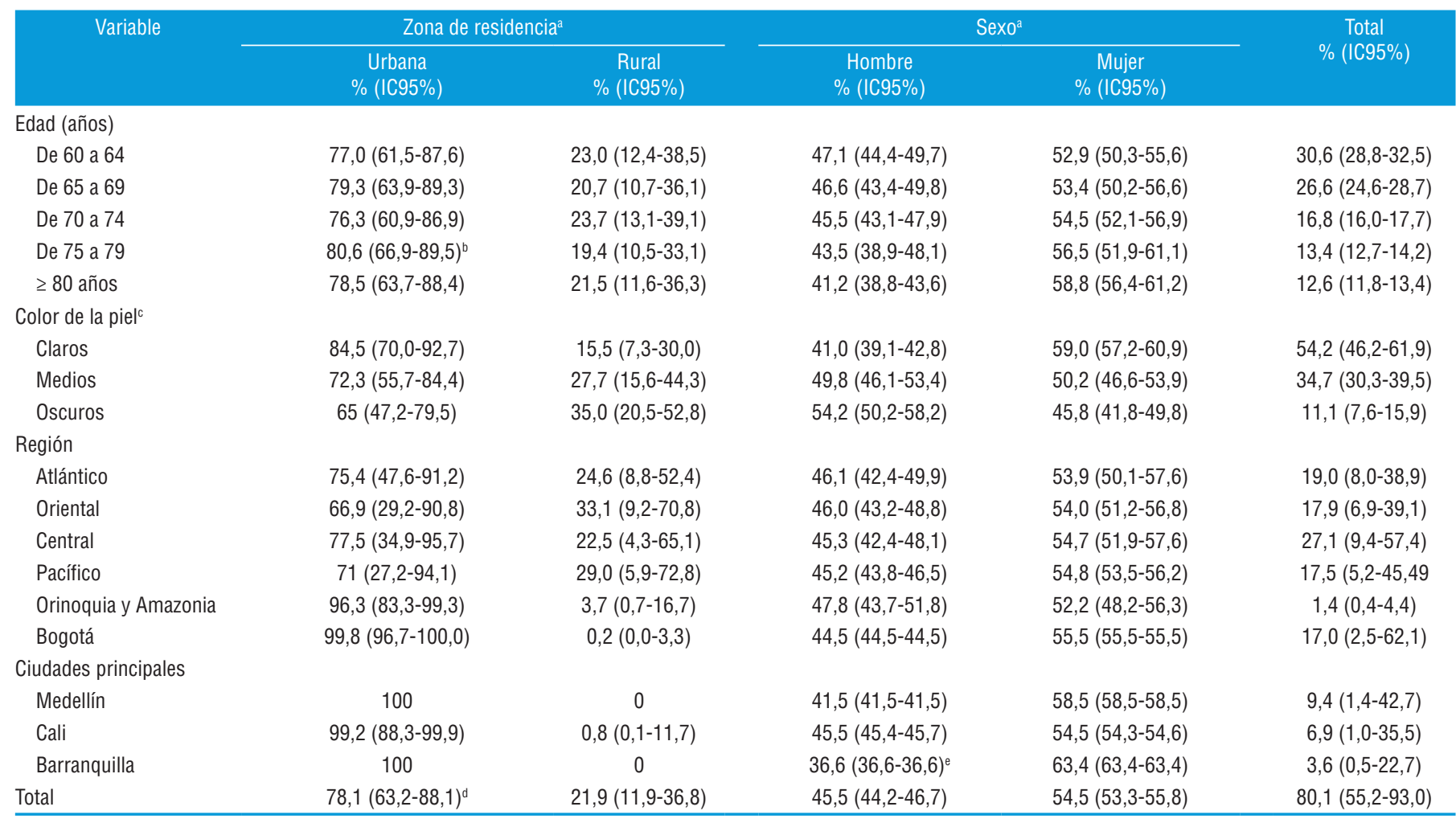

Fuente: elaboración por los autores.

confianza de $95 \%$.

Se comparan los valores de las dos categorías (urbana vs rural, y hombre vs mujer).

列

"Se aprecia un porcentaje significativamente mayor de personas de color claro en las zonas urbanas; el mayor porcentaje de las personas que viven en zonas rurales se catalogan como oscuros. Se observó una diferencia significativa en el

porcentaje de hombres y mujeres de piel oscura.

eDe todas las regiones del país, en Barranquilla se presenta un mayor porcentaje de mujeres mayores de 60 años que de hombres de ese grupo de edad; con una diferencia estadísticamente significativa.

atención por parte de su servicio de salud y el 96,2\% no estaban preocupados por recibir maltrato. Según el índice de Barthel, el $84,2 \%$ eran independientes en el momento de la entrevista y solo el 7,4\% utilizaba las tecnologías de la información y las comunicaciones.

Aunque el 42,7\% informó no tener depresión, el 51,7\% presentaba síntomas depresivos, según la escala de Yesavage para evaluar la depresión en personas mayores. La gran mayoría recibía afecto y compañía de sus hijos (78,5\%), convivientes $(92,9 \%)$ y amigos $(89,5 \%)$.

Una proporción significativamente mayor de los encuestados solo terminó la primaria (59\%; IC95\%: 58,3-59,7), en comparación con los que tenían un nivel educacional de secundaria (17,1\%; IC95\%: 16,6-17,7), y tecnológico o universitario (7,5\%; IC95\%: 6,9-8,1); del total, 16,2\% (IC95\%: 15,7-16,7) declaró no tener estudios; la proporción de personas que terminó la primaria fue significativamente mayor que la de los otros grupos.

\section{Índice de envejecimiento activo}

Los IEA calculados para los diferentes dominios analizados, según los datos de la encuesta realizada en el 2015 y las ponderaciones porcentuales aplicadas, estuvieron entre 4,0 y 17,4. La sumatoria de los IEA ponderados de los cuatro dominios dio como resultado el IEA nacional, que fue 37,4 (cuadro 1). Dentro del dominio actividades remuneradas, todos los grupos mostraron altos porcentajes de actividades relacionadas con el empleo, lo que elevó el índice de este dominio y el índice general. En cuanto a la participación social — parte del dominio actividades no remuneradas-, las actividades de mayor peso fueron el cuidado de los nietos $(14,8)$ y la participación social $(9,3)$, principalmente en grupos religiosos, de salud y de personas mayores. En el dominio vida independiente y activa, cabe resaltar el alto porcentaje de personas mayores independientes según el índice de Barthel $(84,2 \%)$.

De las cuatro variables construidas a partir de los datos que suministra el DANE, la proporción de personas mayores de 65 años que fungen como cabeza de hogares que se encuentran por encima del nivel de pobreza - perteneciente al dominio vida autónoma e independiente- fue $87,4 \%$, aunque la proporción de personas con 60 años o más con ingresos superiores al promedio per cápita en Colombia fue muy bajo (0,3\%). Las otras dos variables - pertenecientes al dominio entorno propicio y saludable para el envejecimiento activo- fueron la proporción de la esperanza de vida alcanzada sobre el objetivo de vivir 105 años, con $0,40 \%$, y la proporción de la esperanza de vida saludable después de los 60 años sobre la esperanza de vida a los 60 años, con $0,034 \%$. 


\section{DISCUSIÓN}

En Colombia -y posiblemente en una gran parte de los países de América Latina-, aún se considera a la vejez como una etapa de la vida con poca participación en la sociedad, caracterizada por tener múltiples enfermedades y un aumento considerable de la discapacidad; esa percepción obstaculiza el proceso de optimización de las oportunidades de las personas mayores $(15,16)$.

El puntaje del IEA estimado en este trabajo se encuentra ligeramente por encima del valor de 35,8\% calculado en promedio para Europa, con países que tienen economías mucho más fuertes que Colombia (17). Esta circunstancia ratifica que el factor económico no es el único condicionante de una vejez activa y saludable.

El análisis por dominios muestra una alta empleabilidad de las personas mayores en Colombia, a pesar de que la encuesta $\mathrm{SABE}$ no toma en cuenta el tipo de trabajo desempeñado. De acuerdo con cifras de la Organización Panamericana de la Salud, el 85,0\% de las personas mayores en Colombia están asociadas a trabajos informales (18), lo que se refleja en que el 90,6\% de los encuestados ganan el equivalente a un salario mínimo vigente $o$ menos, y que solo el $28,5 \%$ de las personas mayores reciben una pensión (11). Esto indica que la empleabilidad en Colombia no está relacionada con mejores condiciones económicas y de vida para sus personas mayores.

El dominio actividad en el empleo no mide su calidad, solo relaciona si se tiene o no empleo en forma general, por lo que la empleabilidad observada en estas circunstancias puede no reflejar mayores niveles de vulnerabilidad y riesgo de pobreza (19). Llama la atención que el $67,4 \%$ de las personas mayores afiliadas al sistema contributivo de salud no tienen una actividad remunerada, algo que podría deberse a la alta proporción de ellos que mantienen relaciones con sus hijos, convivientes y amigos; para explicar este hecho, sin embargo, se requieren estudios específicos que permitan un análisis más profundo.

Al analizar el dominio participación social, se observó que se dedica tiempo a actividades que requieren unión y contacto con los demás, lo que ayuda a mantener un adecuado entramado social (20). En general, la principal actividad declarada está relacionada con el cuidado de los nietos, mientras que otras actividades, como el voluntariado, son menos frecuentes. Dentro de los grupos de participación social, el mayor porcentaje se concentra en la asistencia a grupos religiosos (alrededor del 30\%); la participación en grupos políticos y gremiales está por debajo del $5 \%$. Aunque el cuidado de los hijos o nietos puede considerarse una actividad importante y de impacto social $(5,7)$, la baja participación en asuntos relacionados con el desarrollo de la sociedad y el país reduce las posibilidades de este grupo de la población de alcanzar niveles adecuados de envejecimiento activo.

En el dominio vida autónoma e independiente, pocas personas mayores encuestadas $(20,8 \%)$ manifestaron practicar activamente deportes o ejercicios que, según las recomendaciones, deben realizarse tres veces por semana y catalogarse como vigorosos. No obstante, este porcentaje es mayor que el de otros países, como México y España (19). Comparativamente, caminar tres veces por semana resultó más frecuente en las personas encuestadas $(52,5 \%)$, lo que concuerda con el buen estado funcional que presentan.

Los altos porcentajes observados de recibir atención médica y odontológica cuando se solicitó pueden estar relacionados con los altos niveles de cobertura del sistema de salud en Colombia (21).

El indicador de la seguridad financiera — traducido en renta media y riesgo de pobreza- fue bajo, ya que $90,6 \%$ de las personas mayores encuestadas ganaban solo un salario mínimo mensual o menos. Aunque 87,4\% de las personas mayores de 65 años que fungían como cabeza de hogares lo hacían en hogares que se encontraban por encima del nivel de pobreza, según datos del DANE, 5,9\% de los hogares encabezados por personas de 65 años o más se encuentran en pobreza absoluta $(14,22)$. Este panorama muestra la alta vulnerabilidad de este grupo de la población.

La proporción de personas mayores vinculadas a actividades de aprendizaje en algún establecimiento de educación fue muy baja $(0,9 \%)$, lo que limita considerablemente la posibilidad de adquirir nuevas habilidades y competencias, y de modificar actitudes $(23,24)$, algo de gran valor para mejorar la cohesión social, la vida activa y la participación en el mercado laboral.

El dominio capacidad y entorno propicio para el envejecimiento activo mostró un comportamiento favorable gracias a un mayor nivel de contacto con familiares y amistades. Por otro lado, la expectativa de vida para los mayores de 60 años ha aumentado en dos décadas, lo que coincide con la disminución de la tasa de fecundidad y la disminución de la tasa de mortalidad (22).

Según los resultados de aplicar la escala de Yesavage para evaluar la depresión en personas mayores, el 5,6\% tenía depresión, aunque un elevado número de personas (51,7\%) presentaba algún síntoma depresivo (datos no mostrados); este es un porcentaje alto si se compara con lo informado en otros países (25). A esto contribuye el muy bajo uso de las tecnologías de comunicación. El nivel educativo medido es bajo: la mayoría de las personas mayores de Colombia han cursado solo la educación básica primaria y, en promedio, tienen una escolaridad de 5,8 años (24). Esta situación abre la oportunidad para mejorar este indicador mediante intervenciones y acciones apropiadas.

$\mathrm{Al}$ analizar estos resultados se deben tener en cuenta algunas limitaciones. En primer lugar, la encuesta SABE no proporciona todos los datos necesarios para la construcción del IEA según se diseñó para Europa, por lo que se formaron cuatro variables a partir de otra base de datos nacional colombiana. Además, por su conceptualización, el IEA europeo no es fácilmente adaptable a las mediciones hechas para países latinoamericanos, por lo que la interpretación dependerá de las individualidades especiales en cada país, situación de la que no escapa el índice calculado aquí para Colombia. Por último, los resultados se basan en los datos de una encuesta realizada en el año 2015, por lo que pueden no reflejar fielmente la situación de la población de 60 años o más en Colombia al día de hoy, especialmente si se toman en cuenta los cambios producidos en el último año por la pandemia de COVID-19.

No obstante, este estudio presenta fortalezas, ya que es, hasta el momento, el primero en abordar la situación del envejecimiento activo en Colombia según los parámetros establecidos para el cálculo del IEA. Este índice es una medida comparativa internacionalmente y se define como "una herramienta para evaluar el potencial que tienen las personas mayores para envejecer activa y saludablemente [que] mide el nivel en que estas desarrollan vidas independientes, participan en actividades sociales, laborales y su capacidad para envejecer activamente" 
(10), a diferencia de otras medidas, como el índice global de envejecimiento Agewatch 2015, que evalúa solo los factores que determinan "el bienestar social y económico de las personas mayores en el mundo" (26).

\section{CONCLUSIONES}

Con el IEA calculado se llega a una primera aproximación diagnóstica real de la situación de las personas mayores en Colombia y sus posibilidades de transitar por un envejecimiento activo. A pesar de que más de la mitad de los encuestados carecían de educación elemental o tenían un bajo nivel de escolaridad, solo un bajo porcentaje de ellos trataba de mejorarlo, lo que podría estar relacionado con los bajos ingresos y que terminaran dedicándose a labores como el cuidado de nietos y familiares. Se requiere investigar más profundamente las causas y las vías para mejorar esta situación.

Se deben modificar las políticas públicas para cerrar las brechas identificadas y elevar la calidad de vida de las personas mayores mediante la oferta de mejores oportunidades que les permitan optar por una adecuada remuneración y una mejor calidad de vida. Se debe cambiar de una visión de servicios centrados en la enfermedad a nuevos enfoques basados en la promoción de la salud y la prevención de la discapacidad.

Contribución de los autores. Todos los autores concibieron el estudio original, planificaron la adecuación de las variables desde las bases de datos secundarias, analizaron e interpretaron los resultados, y escribieron el manuscrito. Todos los autores revisaron y aprobaron la versión final.

\section{Conflictos de intereses. Ninguno.}

\section{Financiación. Ninguna.}

Declaración. Las opiniones expresadas en este artículo son responsabilidad de los autores y no reflejan necesariamente los criterios ni la política de la Revista Panamericana de Salud Pública / Pan American Journal of Public Health y/o de la Organización Panamericana de la Salud.

\section{REFERENCIAS}

1. Organización Mundial de la Salud. Informe Mundial sobre el Envejecimiento y la Salud. 2015. Ginebra: OMS; 2015.

2. Organización Mundial de la Salud. Perfiles y tendencias. Salud y envejecimiento: un documento para el debate sobre personas mayores. Madrid: Ministerio del Trabajo y Asuntos Sociales de España; 2001.

3. Organización Mundial de la Salud. Envejecimiento activo: un marco político. Rev Esp Geriatr Gerontol. 2002;37(S2):74-105 [acceso el 10 de noviembre del 2020]. Disponible en: https://ccp.ucr.ac.cr/bvp/ pdf/vejez/oms_envejecimiento_activo.pdf

4. Dayana K, Daza R. Vejez y envejecimiento. Bogotá: Universidad del Rosario; 2011. (Serie Documentos de la Escuela de Medicina y Ciencias de la Salud) [acceso el 12 de noviembre del 2020]. Disponible en: http:/ / repository.urosario.edu.co/handle/10336/3286

5. Kalache A. Envejecimiento activo: un marco político ante la revolución de la longevidad. 1. ${ }^{a}$ ed. Río de Janeiro: Escuela Andaluza de Salud Pública; 2015.

6. Montes de Oca V. El envejecimiento en el debate mundial: reflexión académica y política. Papeles Poblac UNAM. 2003;9(35):77-100.

7. Ramos Monteagudo AM, Yordi García M, Miranda Ramos MA. El envejecimiento activo: importancia de su promoción para sociedades envejecidas. Rev Arch Medicos Camagüey. 2016;20(3):330-7 [citado el 10 de noviembre del 2020]. Disponible en: http://scielo. sld.cu/pdf/amc/v20n3/amc140306.pdf

8. Comisión Económica para América Latina y el Caribe. Informe de la Cuarta Conferencia Regional Intergubernamental sobre Envejecimieto y Derechos de las Personas Mayores en América Latina y el Caribe. Santiago: CEPAL; 2017 [citado el 12 de noviembre del 2020]. Disponible en: https://repositorio.cepal.org/bitstream/ handle $/ 11362 / 42238 /$ S1700921_es.pdf?sequence $=1 \&$ isAllowed $=y$

9. República de Colombia, Ministerio de Salud y Protección Social. Politica Colombiana de Envejecimiento Humano y Vejez 20142024. Bogotá: MSPS; 2015 [citado el 10 de noviembre del 2020]. Disponible en: https://www.minsalud.gov.co/sites/rid/Lists/ BibliotecaDigital/RIDE/DE/PS / Pol\%C3\%ADtica-colombianaenvejecimiento-humano-vejez-2015-2024.pdf

10. Zaidi A, Gasior K, Hofmarcher MM, Lelkes O, Marin B, Rodrigues $\mathrm{R}$, et al. Active ageing index 2012. Concept, methodology and final results. Vienna: European Centre; 2012 [citado el 10 de noviembre del 2020]. Disponible en: https:/ / www.euro.centre.org/ publications/detail/370

11. República de Colombia, Ministerio de Salud y Protección Social, Departamento Administrativo de Ciencia, Tecnología e Innovación. Encuesta SABE Colombia: situación de Salud, Bienestar y
Envejecimiento en Colombia. Colciencias. 2016;476 [citado el 10 de noviembre del 2020]. Disponible en: https://www.minsalud. gov.co/sites/rid/Lists/BibliotecaDigital/RIDE/VS/ED/GCFI/ Resumen-Ejecutivo-Encuesta-SABE.pdf

12. Edward P, Steele L. Pigmentocracia en las Américas: ¿cómo se relaciona el logro educativo con el color de la piel? Nashville, TE: Proyecto de Opinión Pública de América Latina; 2012 (Serie Perspectives desde el Barómetro de las Américas No. 73).

13. Mahoney FI, Barthel DW. Functional evaluation: The Barthel index. Md State Med J. 1965;14:61-5 [citado el 21 de abril del 2021]. Disponible en: https:/ / pubmed.ncbi.nlm.nih.gov/14258950/

14. República de Colombia, Departamento Administrativo Nacional de Estadística. Proyecciones de población 2005-2020, Colombia. Tablas abreviadas de mortalidad nacionales y departamentales 1985-2020. Bogotá: DANE: 2015.

15. Curcio Borrero CL. Investigación y envejecimiento: del dato a la teoría. Hacia Promoc Salud. 2010;15(1):144-66.

16. Módenes Franco P, Sánchez Cabaco A. To know how to age: positive aspects and new perspective. Foro Educacion. 2018;6(10):369-83.

17. Lamura G, Principi A, Di Rosa M. Índice de envejecimiento activo 2018: informe analítico. Ginebra: Comisión Económica de las Naciones Unidas para Europa; 2019 [citado el 10 de noviembre del 2020]. Disponible en: https://www.researchgate.net/publication/ 336899207_2018_Active_Ageing_Index_-_Analytical_Report

18. Fondo de Población de las Naciones Unidas. Una mirada sobre el envejecimiento ¿Dónde están varios países latinoamericanos a 15 años del Plan de Acción Internacional de Madrid? Informe técnico. Ciudad de Panamá: UNFPA; 2017 [citado el 12 de noviembre del 2020]. Disponible en: https://gerontologia.org/portal/archivos Upload/uploadManual/Unamiradasobreelenvejecimiento.pdf

19. Alonso Álvarez M, González González C. Estudio comparativo de la calidad de vida de los adultos mayores entre México y España. VIII Congreso de la Asociación Latinoamericana de Población. Población y desarrollo sostenible: Políticas públicas y avances en la medición sociodemográfica, Puebla, Puebla, México, 2018 23-26 de octubre [citado el 10 de noviembre del 2020]. Disponible en: http:/ / www.alapop.org/Congreso2018/PDF/Poster/00055.pdf

20. Causapié P, López-Cerón A, Porras M, Mateo A. Envejecimiento activo. Libro Blanco. 1. ${ }^{\mathrm{a}}$ ed. Madrid: Ministerio de Sanidad y Protección Social; 2011.

21. Ayala J. La salud en Colombia: más cobertura pero menos acceso. Bogotá: Banco La República; 2014. (Documento de trabajo sobre economía regional 2014). 
22. República de Colombia, Ministerio de Salud y Protección Social, Oficina de Promoción Social. Sala situacional de la población adulta mayor. Bogotá: MSPS; 2018 [citado el 10 de noviembre del 2020]. Disponible en: https://www.minsalud.gov.co/sites/rid/Lists/ BibliotecaDigital/RIDE/DE/PS/sala-situacion-envejecimiento2018.pdf

23. Muñoz F, Manuel J, Almendro E. Envejecimiento activo y desigualdades de género. Aten Prim (Madrid): 2008;40(6):305-9. Disponible en: https:/ / www.elsevier.es/es-revista-atencion-primaria27-pdf-13123684

24. República de Colombia, Observatorio de la Democracia. La situación de los adultos mayores en Colombia. Bogotá: Observatorio de la Democracia; 2017 [citado el 10 de noviembre del 2020]. Disponible en: https://obsdemocracia.org/publicaciones/noticias/ la-situacion-de-los-adultos-mayores-en-colombia/
25. Fernández-Ballesteros R. Positive ageing : Objective, subjective and combined outcomes. Electronic J Applied Psychol. 2011;7(1):22-30.

26. Scobie J, Asfour L, Beales S, McGeachie P, Gillam S, Mihnovits A, et al. Indice global de envejecimiento, AgeWatch 2015. Informe a profundidad. Londres: HelpAge International; 2015 [citado el 18 de febrero del 2021]. Disponible en: www.helpage.org info@ helpage.2015;28

Manuscrito recibido el 2 de diciembre del 2020. Aceptado para publicación, tras revisión, el 12 de marzo del 2021.

\section{Active Aging Index in Colombia: analysis based on the national survey of health, well-being, and aging (SABE Colombia 2015)}

ABSTRACT Objectives. To describe the current state of the population aged 60 years and older in Colombia and to determine the Active Aging Index (AAl) and assess its domains.

Methods. Based on data collected during the national survey of health, well-being, and aging conducted in Colombia in 2015 (known as SABE Colombia 2015), the AAl for Colombia was calculated using the methodology applied in the countries of the European Union, with the same four domains. The methodology was adapted to the data provided by the survey and to the Colombian context.

Results. The AAI for Colombia was 37.4. Older adults in Colombia had a high level of employability, but the largest percentage of jobs was in the informal sector. A small percentage of older adults were active in political groups, associations, or organizations advocating for decisions that will benefit society and the country. The fact that a large majority of older adults in Colombia are able to function adequately presents opportunities to improve their performance in line with the determinants of active aging.

Conclusions. This study is the first real attempt to characterize the state of older adults in Colombia and their possibilities to achieve active aging. Public policies should be adjusted to close the identified gaps and improve the quality of life for older adults by offering opportunities for them to earn adequate pay for a better quality of life. The service-centered view of illness should be shifted to focus more on new approaches that include health promotion and prevention of disability.

Keywords $\quad$ Aged; healthy aging; Colombia. 


\section{Índice de envelhecimento ativo na Colômbia: uma análise a partir da Pesquisa Nacional de Saúde, Bem-estar e Envelhecimento (SABE da Colômbia 2015)}

RESUMO Objetivos. Descrever a situação atual da população com 60 anos ou mais de idade na Colômbia e determinar o índice de envelhecimento ativo (IEA) e seus domínios.

Métodos. O IEA e seus quatro domínios foram calculados a partir de dados coletados na Pesquisa Nacional de Saúde, Bem-estar e Envelhecimento realizada na Colômbia em 2015 (SABE Colômbia 2015). Este cálculo se baseou em metodologia elaborada para os países da União Europeia, com a adaptação dos domínios de acordo com os dados do levantamento e o contexto colombiano.

Resultados. O IEA da Colômbia é de 37,4. Observou-se alta empregabilidade entre os idosos colombianos, mas os empregos são na sua maioria de caráter informal. Um pequeno percentual dos idosos se ocupa de atividades em agremiações políticas ou sindicais ou em grupos que tomam decisões com repercussão social e para o país. A grande maioria dos idosos na Colômbia tem níveis adequados de funcionalidade, com potencial de melhorar seu desempenho segundo os determinantes de envelhecimento ativo.

Conclusões. Trata-se do primeiro diagnóstico real da situação do idoso na Colômbia e suas possibilidades de envelhecimento ativo. As políticas públicas precisam ser modificadas para reduzir as lacunas identificadas e melhorar a qualidade de vida dos idosos, oferecendo a eles mais oportunidades de exercer atividades com remuneração adequada e ter uma melhor qualidade de vida. É necessária uma nova visão, mudando o enfoque de serviços centrados na doença para novas abordagens baseadas na promoção da saúde e prevenção de incapacidade. 